\title{
EFECTO DE LA UTILIZACIÓN DE AGREGADOS DE CONCRETO RECICLADO SOBRE EL AMBIENTE Y LA CONSTRUCCIÓN DE VIVIENDAS EN LA CIUDAD DE HUAMACHUCO
}

\author{
EFFECT OF THE USE OF RECYCLED CONCRE'TE AGGREGATES ON \\ THE ENVIRONMENT AND HOUSING CONSTRUCTION IN \\ HUAMACHUCO CITY
}

Jorge Wilmer Elías Silupu ${ }^{*}$, (D) Jorge Enrique Flores Franco ${ }^{2}$, (D) Ramon Enrique Barrera Gutiérrez ${ }^{2}$, Cesar Alberto Reyna Pary ${ }^{3}$

jelias_s@hotmail.es; jfloresf@gmail.com; rebagu@hotmail.com; reynapary@hotmail.com

${ }^{1}$ Universidad Nacional Ciro Alegría, La Libertad, Perú

${ }^{2}$ Universidad Nacional de Trujillo, La Libertad, Perú

${ }^{3}$ Municipalidad Provincial de Huamachuco, La Libertad, Perú

*Correspondencia: Jorge Wilmer Elías Silupu. Email: jelias_s@hotmail.es

Recibido: 28.01.2020 | Aprobado: 12.03.2020

\section{RESUMEN}

En la presente investigación se logró determinar el efecto de utilizar los agregados de concreto reciclado (ACR) como materia prima en la elaboración de concreto para la construcción de viviendas en la ciudad de Huamachuco. Se utilizó como materia prima, para el diseño de mezclas, el cemento Portland Tipo I, gravilla de 1/2" y 3/4", arena gruesa y los agregados de concreto reciclado, estos últimos sustituyeron a la arena gruesa y a la gravilla en los porcentajes en volumen de $50 \%, 75 \%$ y $100 \%$ respectivamente. Se elaboraron probetas de concreto simple y concreto conteniendo ACR según la Norma Técnica Peruana 339.033, luego se realizó el ensayo de compresión a las probetas, después de 7, 14 y 28 días de curado, según la Norma ASTM C39, así se pudo determinar que el concreto conteniendo 50\% ACR presento la mejor resistencia a la compresión, alcanzando $200,18 \mathrm{~kg} / \mathrm{cm}^{2}$. Además, se pudo observar que la resistencia a la compresión del concreto conteniendo 100\% ACR después de 14 días presenta una tendencia casi lineal. Finalmente, se elaboró el inventario para el análisis del ciclo de vida (ACV) del concreto, dicha información nos servirá para realizar el ACV del concreto empleando para tal fin el software libre OPENLCA.

Palabras clave: Concreto reciclado, resistencia a la compresión, análisis del ciclo de vida.

\section{ABSTRACT}

In the present research was determined the effect of using the recycled concrete aggregates (RCA) as raw material in the elaboration of concrete for the construction of houses in the city of Huamachuco. Portland cement Type I, 1/2 "and 3/4" gravel, coarse sand and aggregates of recycled concrete were used as raw material for the design of mixtures, these last replacing coarse sand and gravel in the percentages by volume of $50 \%$, $75 \%$ and $100 \%$ respectively. Specimens of simple concrete and concrete containing RCA were prepared according to Peruvian Technical Standard 339.033, then the compression test was performed on the specimens after 7, 14 and 28 days of curing, according to ASTM C39, so the concrete containing 50\% RCA showed the best compressive strength, reaching $200.18 \mathrm{~kg} / \mathrm{cm}^{2}$. Also, it was observed that the compressive strength of the concrete containing 100\% ACR after 14 days presents an almost linear tendency. Finally, the inventory for the life cycle assessment (ACV) of the concrete was elaborated, this information will serve to realize the ACV of the concrete using for that purpose the OPENLCA free software.

Keywords: Recycled concrete, compressive strength, life cycle assesment. 


\section{INTRODUCCIÓN}

Los elementos para la construcción a través de la historia son muchos; desde el lodo, las rocas y otros muchos recursos o productos que con aciertos y errores se han ido poniendo en uso. En la actualidad, el más usado es el concreto. Dado que la demanda por materiales de construcción es elevada y sobre todo de calidad, el presente trabajo toma como propuesta la incorporación de residuos sólidos reciclados de concreto, remplazando de completo o en alguna medida el agregado grueso, grava, para darle una proporción similares propiedades a las diferentes concreto simples y buscar su mejora en ciertos aspectos. Es así como se daría el cuidado del medio ambiente, en el marco del principio de desarrollo sostenible, y buscando el cumplimiento de los requisitos de calidad y durabilidad al servicio de la sociedad en construcción de habitaciones.

Durante mucho tiempo, todo lo que se realiza lo hacemos pensando en el cuidado del medio ambiente. Hoy en día es conocida la problemática que sucede en el planeta, sobre todo cuando hacemos uso sin tener ninguna conciencia ambientalista sobre uso desmedido de los recursos naturales que día a día están acabando con nuestro planeta.

Los constantes cambios climáticos que van originando cada vez más devastadores fenómenos naturales, nos están llevando a tomar conciencia, para el uso adecuado de nuestros recursos naturales y que hoy en día en pleno siglo XXI, nos están llevando a doctor métodos como el reciclaje, reúso y sustitución de materias primas naturales, por otras que no afectan las condiciones terrestres. Otras de las cosas que no podemos dejar de mencionar crecimiento exponencial de la población mundial y local. Aparte de estar acabando con las zonas naturales, generando gran consumo de materia primas en la construcción de viviendas en las zonas rurales y urbanas. A continuación, se mencionan antecedentes relacionados con esta investigación.

El material de concreto para la construcción es hoy en día la más utilizada por las compañías, se estima que alrededor de unos 25 millones de toneladas de concreto se producen a nivel mundial cada año, lo cual viene hacer aproximadamente 3,8 toneladas por persona al año. Lo cual es dos veces más el concreto usado en la construcción en todo el planeta, como todos los demás materiales de construcción juntos. Además de la cantidad de residuos generados al año en Europa, alrededor del 40\% proviene de la construcción y los residuos de demolición (Marinković et al., 2015).

El concreto demolido puede ser reciclado, a pesar de que no se puede reciclar de nuevo sus materiales constituyentes originales o toda forma original. Más bien, el concreto es triturado convertido en agregado llamado agregado de concreto reciclado (Marinković et al., 2015).

El reciclaje de concreto para la fabricación de agregados gruesos y sustituir al natural debe ser una práctica que debe empezar a realizarlo a la brevedad posible ya que se encuentra disponible de los bancos de materiales pétreos lo cual es cada día más escasos (Martínez y Mendoza, 2005).

Los agregados reciclados con una granulometría adecuada producen mezclas de buena calidad y con unas pruebas mecánicas similares al de concreto natural. El concreto 
reciclado puede ser utilizado como concretos de clase dos, lo cual lo convierte en un concreto de calidad con una aplicación nada despreciable (Martínez y Mendoza, 2005).

Los agregados de concreto reciclado de la demolición constituyen uno de los flujos de residuos más grandes dentro de los países desarrollados. El uso de agregados alternativos establecidos a partir de materiales de desecho podría ser un paso hacia la solución de parte del agotamiento de los agregados naturales. La extracción abusiva de los agregados desde los recursos naturales se ha destacado a nivel internacional, debido a la disminución de la cantidad de recursos primarios en el contexto de una conciencia de la protección del medio ambiente. El campo de la construcción es responsable de los flujos considerables de residuos dentro de la sociedad humana, así como para el agotamiento de materiales y energía consumidos. El agregado de concreto reciclado utilizado para la construcción puede aliviar el problema de la escasez de agregados y reducir tanto la contaminación ambiental y la huella ecológica (Serres et al., 2015).

La necesidad de reciclar los residuos de construcción no solamente concierne a las los países industrializados, sino que también a los semi desarrollados que presentan una demanda global con más prioridades.

En muchos países, que desde los mas industrializados tales como Holanda o Dinamarca, u otros países en vías de desarrollo como es el Perú, experimentalmente a partir de las buenas prácticas ambientales se podrá tener un ahorro de recursos naturales. desde el punto de vista ambiental, el reciclaje de escombro es bastante atractivo por que aumenta la vida útil de los rellenos sanitarios y los botaderos de cielo abierto para luego evitar la degradación de los recursos naturales no renovables; pero desde punto de vista económico, el concreto reciclado resulta muy atractivo cuando el producto es competitivo con otros materiales con relación al costo y la calidad del producto (Bedoya C., 2003).

Los agregados que proviene de la recuperación producidas en la construcción de edificios y residuos de demolición son reutilizados como sustitutos de los agregados de arena natural en la fabricación adoquines de concreto. Un "análisis del ciclo de vida" de los elementos particulares fue llevado a cabo, para determinar el consumo energético de todos los procesos que intervienen con el propósito de llegar al producto final. Muchos estudios sobre el procedimiento de tratamiento de residuos que se han realizado en las últimas décadas, pero no muchos con aplicaciones en la industria del concreto (López, et al., 2015).

Se ha demostrado que es posible la fabricar bordillos de una sola capa con agregados reciclados, sin ningún efecto negativo en sus propiedades mecánicas si el porcentaje de sustitución es 50\% o menos. Sin embargo, un estudio adicional de la durabilidad y vida útil reafirmaría este tema (López et al., 2015).

Uno de los grandes desafíos ambientales en la industria de la construcción es una fuerte fuerza social para disminuir el consumo a granel de los materiales de construcción en el entorno urbano. Teniendo en cuenta estos precedentes las aplicaciones de más tecnologías del reciclado in situ de residuos de la construcción y demolición (RCD) es una necesidad urgente (Lotfi et al., 2015). 
Con el fin de reducir el impacto ambiental de los residuos de construcción y de demolición, un nuevo proceso de reciclaje de concreto en el marco del proyecto europeo C2CA se ha desarrollado. El objetivo está en el reciclado mecánico in situ del concreto EOL con agregado de alta calidad y materia prima con baja emisión de $\mathrm{CO}_{2}$ para la producción del Clinker (Lotfi et al., 2015).

La sustitución de más del 50\% del agregado grueso con concreto reciclado - que corresponde a la utilización de más de $500 \mathrm{~kg}$ de RA por $\mathrm{m}^{3}$ de concreto nuevo - necesita hacerse más cuidadosamente y las aplicaciones deben limitarse a las condiciones de exposición leves (Lotfi et al., 2015).

El desarrollo en muchos sectores tiene efectos ambientales negativos. En el sector de la construcción, existen millones de toneladas generadas por las empresas de las mismas conocidos como residuos de construcción y demolición (RCD) en cada año. Estos RCD originan un daño significativo en el ambiente y puede poner en peligro la SOSTENIBILIDAD. Para poder encontrar una solución y poder preservar los recursos naturales, en particular los recursos no renovables, las investigaciones en la mayoría de todo el mundo sobre los áridos reciclados que se han incrementado en su mayoría con el fin de investigar sus posibilidades con su valorización con el concreto (Omary et al. 2016).

Los RCD puede cubrir una amplia gama de materiales, que dependen de sus orígenes: la demolición total o parcial de la infraestructura, la construcción de edificios, la excavación para nivelación de tierras, obras civiles y/o cimentaciones generales y las actividades de mantenimiento de carreteras. Se estima que unos 900 millones de toneladas de RCD se generan cada año en Europa, EE.UU. y Japón según lo informado por el Consejo Mundial Empresarial para el Desarrollo Sostenible (Omary et al. 2016).

La sostenibilidad es una preocupación apremiante del siglo 21. El desarrollo social en la mayoría de los países, es la sensibilización de satisfacción de sus necesidades y las generaciones futuras, con una legislación para proteger la calidad de vida presente y futura. Estas necesidades son apremiantes para diversos sectores, como la construcción, para encontrar soluciones sostenibles. La masificación de la incorporación de agregados reciclados disminuiría el impacto ambiental de la industria del concreto, por ejemplo, reducción de las disposiciones en vertederos, menos extracción en canteras y distancias de transporte más cortas (Brito et al., 2016)

Los resultados de varios experimentos han demostrado que estos agregados gruesos reciclados pueden ser utilizados en el concreto nuevo sin efectos perjudiciales y que las diversas normas que impiden la incorporación de agregados reciclados deben ser revisada (Brito et al., 2016).

Debido al Impacto ambiental que generan los residuos sólidos de construcción y demolición (RCD), el reciclaje es obligatorio de acuerdo a la ley 27314 es importante que los agregados de concreto reciclado (ACR) sean utilizados en el concreto para poder cumplir con la demanda del mercado, se han hecho estudios muy limitados sobre el origen de los residuos y los procesos de transformación influyendo mucho en las características de los agregados de concreto reciclado (Gomes et al., 2015). 
La gestión de los residuos de construcción y demolición como también los residuos de vidrio es una seria preocupación en país de Hong Kong, así como de otros países debido a su naturaleza no combustibles y no putrescible, y que al quedarse sin el medio de eliminación. Sin embargo, se consideran residuos minerales derivados de las actividades de construcción y demolición, el desecho de vidrio que tienen un potencial para ser reutilizado como materiales en la construcción, especialmente en forma de agregados, pero ningún estudio ha tratado de evaluar la sostenibilidad de las técnicas de análisis del ciclo de vida (ACV). Con el fin de aumentar la conciencia ambiental en la industria de la construcción, por lo tanto, es necesaria una evaluación del comportamiento medioambiental de los materiales de construcción mediante un análisis del ciclo de vida (Uzzal et al., 2016).

El objetivo de esta investigación es estudiar la viabilidad de la incorporación de una fracción fina de los agregados reciclados procedentes de los residuos de construcción y demolición en la fabricación de mortero para mampostería a base de cemento. Inicialmente, tres tipos de áridos reciclados como agregados reciclados de concreto, mixtos y de cerámica, que se caracterizaron en términos de propiedades físicas, mecánicas y químicas. Para ello se utilizaron diferentes proporciones de cemento y agregado en 1:5 ,1:75 y 1:1 en porcentajes de sustitución de 50\%, 75\% y 100\% respectivamente para tres tipos de agregados reciclados.

Con los resultados de estudio de las proporciones, los morteros reciclados presentan un comportamiento más pobre que los morteros fabricados utilizando arena natural, sin embargo, cumplió con los límites establecidos por las normas y los fabricantes. Por lo tanto, los resultados de este estudio sugieren la posibilidad de sustituir el 100\% de arena natural con agregados reciclados analizados (Saiz et al., 2016).

Se evaluaron un número de mezclas de concretos verdes que tienen propiedades básicas similares desde el punto de vista del medio ambiente por medio del método de Análisis de Ciclo de Vida, y se compara con una mezcla de concreto convencional correspondiente. Las mezclas de concreto verde investigados se prepararon a partir de tres diferentes tipos de subproductos industriales, es decir, (1) arena de fundición, y (2) las escorias de acero, los cuales fueron utilizados como agregados manufacturados, y (3) las cenizas volantes, que se utilizó como un aditivo mineral. Algunas mezclas de concreto verde también se prepararon a partir de un agregado reciclado, que se obtuvo a partir de residuos de concreto armado. (Turk et al., 2015).

Este artículo nos describe los resultados de un estudio de viabilidad sobre la sustitución parcial del agregado grueso natural con un agregado reciclado de mala calidad como son residuos de construcción y demolición en la fabricación de concreto que tiene como resultado $30 \mathrm{MPa}$ de resistencia. El agregado reciclado mixto utilizado tenía un alto contenido de asfalto y material flotante. La densidad, la resistencia a la compresión y tracción, la absorción de agua y capacidad de adsorción se estudiaron en una serie de hormigones que contienen $25 \%$ o $50 \%$ del material agregado reciclado con y sin materiales flotantes. Las mejoras técnicas, económicas y ambientales derivados de la sustitución potencial del agregado grueso natural con este tipo de residuos de 
construcción y demolición reciclados (RCD), contribuirían a la sostenibilidad de la industria de la construcción (Medina et al. 2014).

Este tipo de agregados reciclados se difieren del agregado natural de acuerdo al primero que contenga mortero de cemento endurecido. Este tipo de montero de cemento adherido sobre los agregados de conconcreto reciclado el cual tiene mayor porosidad y absorción de agua para ello tiene menor resistencia de los agregados naturales. Este tiene efectos negativos sobre las propiedades mecánicas y la durabilidad del concreto fresco y endurecido para ello es hecho con agregados de concreto reciclado. Por lo tanto, facilitará las aplicaciones de, agregado de concreto reciclado si el mortero de cemento adherido se puede mejorar utilizados las proporciones las más indicadas. La Extracción y fortalecimiento del mortero adherido son los dos métodos principales para mejorar las propiedades de los agregados de concreto reciclado (Shi et al. 2016).

El uso adecuado de los recursos naturales es uno de los pilares fundamentales del desarrollo sostenible impuestas a las sociedades modernas. Un uso más eficaz y eficiente de los recursos naturales, así como la mitigación de los impactos ambientales provocados por su extracción podría lograrse si se aplican las políticas de gestión y reciclaje adecuados a los desechos de demolición y construcción. Es necesario la valorización de los residuos de la industria de la construcción y es una forma de conducirnos a sostenibilidad (Silva et al. 2015).

El uso de agregados reciclados (AR) en la construcción constituye un paso significativo hacia una sociedad más sostenible y también crea una nueva oportunidad de mercado para ser explotados. En los últimos años, varios estudios de casos han surgido en el que los agregados reciclados fueron utilizados en aplicaciones geotécnicas, tales como materiales de relleno y en capas de pavimento no unidos (Cardoso et al., 2015).

Las propiedades físicas de los agregados reciclados no sólo dependen del tipo de material reciclado, sino también del proceso de fabricación adoptado. Pruebas estándar de laboratorio son necesarias para evaluar las propiedades de estos materiales, lo que permite una mejor comprensión de su rendimiento cuando se compactan (Cardoso et al., 2015).

El ACV es una metodología innovadora para mejorar la sostenibilidad de la industria en todas las fases del ciclo de vida. La metodología se examinó considerando todas las fases de las normas internacionales ISO 14040 y 14044, explicando en detalle las 4 fases de su aplicación: definición del objetivo y alcance, análisis de inventario (AICV), evaluación de impacto (EICV) y la interpretación de los resultados. (Rodrigues et al, 2016).

Existe una gran necesidad de incorporar los residuos reciclados al inicio del ciclo de vida del concreto, además de la necesidad de transformar los residuos generados al final de su ciclo de vida en insumos para otro sistema de producción o incluso para el sistema de producción del concreto. La necesidad de nuevos estudios de ACV sobre el tratamiento y la reutilización de los residuos de la construcción es evidente para evitar su eliminación en el medio ambiente e incorporarla en el ciclo de vida de los nuevos concretos. Tres factores que deben considerarse al comparar el ACV del concreto común 
con el de concreto ecológico son la selección de la unidad funcional, los datos del inventario y el método EICV (Rodrigues et al, 2016).

El ACV del concreto y sus materias primas sigue siendo un área de investigación limitada a pesar del número creciente de estudios en los últimos años. Los impactos ambientales del ciclo de vida de los materiales distintos del cemento Portland, como las mezclas y el consumo de agua, rara vez se incluyen en los ICV publicados de la producción de concreto. Es necesario seguir investigando las emisiones tóxicas en el aire, además de incluir las emisiones de gases de efecto invernadero y las emisiones de contaminantes atmosféricos. Al igual que los ICV de producción del cemento, los ICV de producción de concreto carecen también de datos que reflejen variaciones en las diferencias tecnológicas y geográficas (Gursel et al, 2016).

La metodología del ACV se empleó para evaluar los impactos ambientales de una planta brasileña de cemento que produce dos tipos de cemento Portland. La etapa de transporte es el mayor contribuyente a todas las categorías de impacto analizadas. Esto ocurre porque el transporte en Brasil se realiza principalmente por carretera, que es el medio de transporte más contaminante. Sin embargo, en el momento de este estudio, la fábrica estaba adquiriendo el clinker de una fuente externa, lo cual no es usual para las industrias del cemento. Como esta condición cambió desde entonces, los pasos más impactantes pueden estar relacionados con la obtención de combustibles fósiles y la clinkerizacion. En este contexto, la sustitución de combustibles fósiles por combustibles alternativos puede ser una opción para alcanzar impactos ambientales más bajos. Sin embargo, las distancias de la fuente de estos combustibles alternativos a la fábrica deben ser consideradas. (Stattford F. et al, 2016).

Se realizó un ACV de cuna a la puerta del concreto ordinario y del concreto reciclado para analizar si el cambio de los datos genéricos a los datos de ACV específicos de los productos produjo diferencias sustanciales y comparar los resultados específicos de la empresa entre el concreto ordinario y el reciclado. Para ello, los impactos ambientales fueron calculados para un concreto ordinario y un concreto reciclado (Ecobeton ${ }^{\circledR}$ ) basado en los datos del fabricante (GCM). Los resultados mostraron que las gravas recicladas tienen menos impactos ambientales en comparación con las gravas vírgenes. Sin embargo, los resultados de $1 \mathrm{~m}^{3}$ de concreto reciclado muestran menos emisiones de gases de efecto invernadero, pero con una diferencia no significativa (inferior al $2 \%$ ) en comparación con el hormigón ordinario. Las diferencias de demanda de energía acumulada son ligeramente superiores (alrededor del 4\%) (kleijer et al, 2017).

\section{MATERIALES Y MÉTODOS}

\section{Materiales}

Para la parte experimental se emplearán los siguientes materiales: Cemento Portland Extra Forte, agregados (piedra chancada y arena gruesa), agregados de concreto reciclado (ACR), recipientes (balde de 15 l), moldes cilíndricos metálicos de 4" x 8", carretilla, cilindros metálicos vacíos, palana, lavadores, babilejo. 


\section{Tratamiento}

Toma de muestras: Las muestras se elaboraron en la ciudad de Huamachuco, teniendo en cuenta las dosificaciones de agregados de concreto reciclado (ACR) al 50\%, $75 \%$ y $100 \%$ en reemplazo de los áridos comunes del concreto. Se elaboraron 3 probetas para cada porcentaje de ACR y 3 testigos de concreto convencional para los ensayos de compresión a los 7, 14 y 28 días respectivamente.

\section{Método}

\section{Ensayo para determinar la Resistencia a la Compresión del Concreto}

Los resultados de la experimentación se realizaron en tres fases; primero en la elaboración de probetas (03 días), segundo en el curado (28 días) y tercero en los ensayos de compresión (03 días).

a. Primero: Se elaboraron las probetas según las proporciones indicadas líneas arriba y siguiendo las indicaciones de la NTP 339.033 (adaptada de la norma ASTM C31).

b. Segundo: Luego de someter las probetas a la etapa de curado, fueron sumergidas en un cilindro conteniendo agua.

c. Tercero: Después de la etapa de curado, se ejecutó el ensayo de compresibilidad.

d. Finalmente se analizaron los resultados obtenidos.

\section{Elaboración del Análisis del Ciclo de Vida del Concreto}

El Análisis del Ciclo de Vida (ACV) es un proceso de varias etapas, cuya definición detallada se da en las normas internacionales de la serie ISO 14040. El ACV tiene como objetivo primordial evaluar las cargas ambientales de un producto o proceso. Las cargas de algunos productos como el concreto se pueden evaluar a través de las diferentes etapas de acuerdo a su ciclo de vida, idealmente de todos ellos. Todas las cargas están asociadas con flujos de materiales y energía (es decir, el uso de recursos naturales y requerimientos de energía) y dependen directamente de las emisiones relacionadas al aire y al agua, con la producción de cualquier tipo de residuos sólidos. En la metodología del ACV es eficaz en el caso de comparaciones de productos, por ejemplo, en los materiales de construcción que difieren en sus materias primas, pero tienen la misma funcionalidad especialmente en este caso el estudio. En tales casos, el ACV puede servir de base para la toma decisiones, teniendo en cuenta el gran interés ambiental para mejorar la sostenibilidad en la industria de la construcción.

En una metodología general del ACV, se considera un conjunto de cuatro etapas o fases, según se muestra en la Figura 1. Cada Fases de este Análisis de Ciclo de Vida; definiremos el objetivo y alcance; el análisis del inventario; y evaluación de impacto. Resulta posible realizar estudios simplificados en donde se trabaje sólo con algunas de las etapas señaladas, dependiendo principalmente de los objetivos del estudio y la información disponible para la aplicación de esta herramienta.

En estos casos y dado que el objetivo principal en la cual se centra la obtención de energía contenida y la huella de carbono (categorías de impacto: consumo energético y emisiones de $\mathrm{CO}_{2}$ ), generados en el proceso de fabricación del concreto, se trabajará con 
un Análisis de Ciclo de Vida Simplificado (ACVs), considerando: definición de objetivos y alcance, análisis de inventario (ICV) e interpretación de resultados.

\section{Figura 1}

Fases del Análisis del Ciclo de Vida.

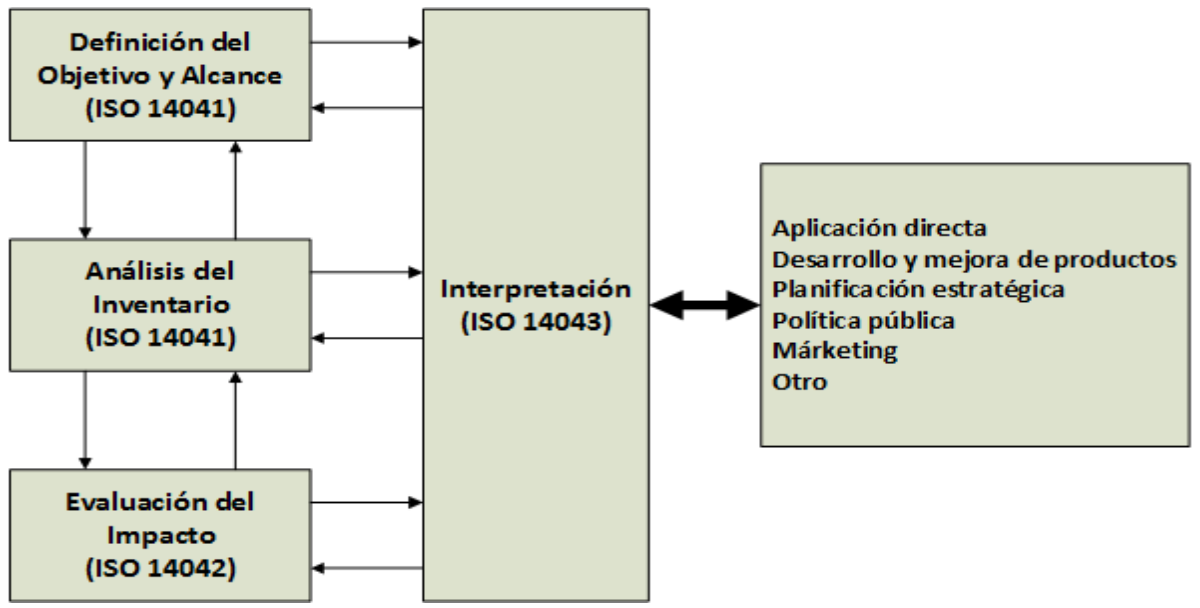

\section{RESULTADOS Y DISCUSIÓN}

Es oportuno indicar que las pruebas experimentales se realizaron empleando el $50 \%, 75 \%$ y $100 \%$ de agregados de concreto reciclado con cemento Portland Tipo I según las dosificaciones que a continuación se detallan:

\section{Tabla 1}

Dosificación en volumen de materiales para la elaboración del concreto.

\begin{tabular}{llll}
\hline \multirow{2}{*}{ Mezcla } & \multicolumn{2}{c}{ Cantidad de materiales en volumen } \\
\cline { 2 - 4 } & Piedra chancada & Arena gruesa & $\begin{array}{l}\text { Agregados de } \\
\text { concreto reciclado }\end{array}$ \\
\hline $0-\mathrm{R}$ & 2 & 2 & 0 \\
$50-\mathrm{R}$ & 1 & 1 & 2 \\
$75-\mathrm{R}$ & 0 & 1 & 3 \\
$100-\mathrm{R}$ & 0 & 0 & 4 \\
\hline
\end{tabular}

Es oportuno indicar que $0-\mathrm{R}$ significa que para la elaboración del concreto no se ha utilizado agregados reciclados, 50 - R significa que se ha utilizado el 50\% de agregados reciclados en la mezcla del concreto y así los demás.

A continuación, se presentan los resultados de las pruebas de resistencia a la compresión del concreto:

\section{Tabla 2}

Resultados de los ensayos de la resistencia a la compresión promedio de las probetas de concreto.

\begin{tabular}{cccc}
\hline \multirow{2}{*}{ Mezcla } & \multicolumn{3}{c}{ Resistencia a la Compresión Promedio f'c (kg/cm2) } \\
\cline { 2 - 4 } & 7 días & 14 días & 28 días \\
\hline $0-\mathrm{R}$ & 158,65 & 196,64 & 217,11 \\
$50-\mathrm{R}$ & 152,89 & 189,13 & 200,18 \\
$75-\mathrm{R}$ & 146,90 & 180,59 & 194,28 \\
$100-\mathrm{R}$ & 141,51 & 176,55 & 185,77 \\
\hline
\end{tabular}


Los resultados mostrados son la resistencia a la compresión promedio de las probetas de concreto que fueron ensayadas a los 7, 14 y 28 días respectivamente.

\section{Figura 2}

Resistencia a la compresión promedio del concreto convencional y del concreto de agregados reciclados.

Resistencia a la Compresión Promedio del Concreto f'c $(\mathrm{kg} / \mathrm{cm} 2)$

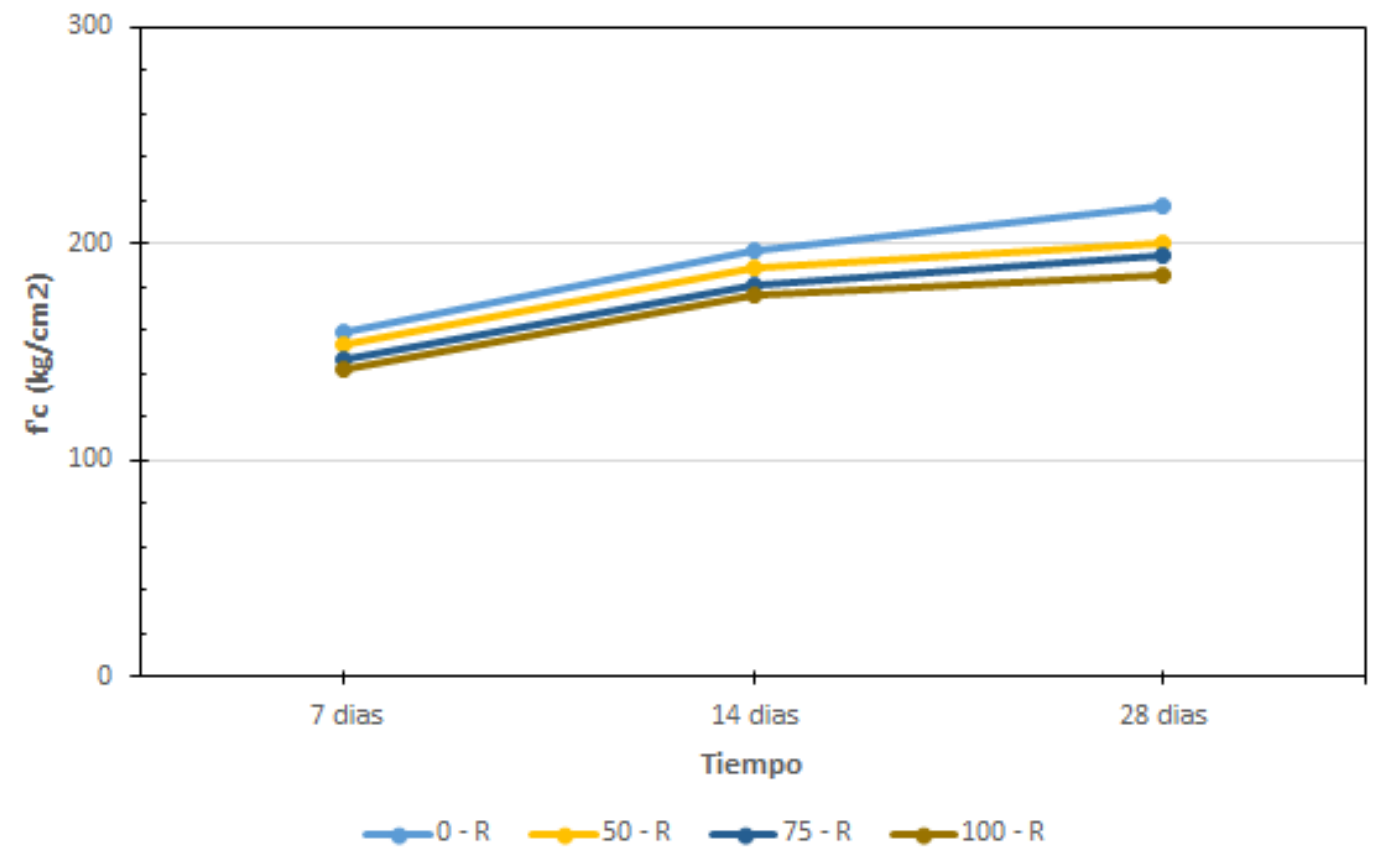

Como se puede observar en la Figura 2, la resistencia del concreto aumenta de manera directamente proporcional con el tiempo para todos los casos, en cuanto al concreto de agregados reciclados el que mostro mejor comportamiento fue el concreto $50-\mathrm{R}$, alcanzando un poco más de $200 \mathrm{~kg} / \mathrm{cm}^{2}$.

También se puede observar la tendencia casi lineal del concreto $100-\mathrm{R}$ a partir de los 14 días de curado.

\section{CONCLUSIONES}

Se ha logrado determinar la resistencia del concreto empleando agregados de concreto reciclado (ACR) en proporciones del 50\%, 75 y 100\%, de los cuales el concreto 50 - R mostró una mejor resistencia a la compresión a los 28 días. Además, se ha observado que la tendencia del concreto con ACR al 50\% ha logrado superar los 200 $\mathrm{kg} / \mathrm{cm}^{2}$ a los 28 días, mientras que el concreto con ACR al $100 \%$ a partir de los 14 días muestra una tendencia casi lineal.

\section{REFERENCIAS BIBLIOGRÁFICAS}

Bedoya-Montoya, C. (2003). El Concreto Reciclado con Escombros como Generador de Hábitats Urbanos Sostenibles. Universidad Nacional de Colombia Sede Medellin, Facultad de Arquitectura, Escuela del Habitat. 
Brito, J., Ferreira, J., Pacheco, J., Soares, D., Guerreiro, M. (2016). Structural, Material, Mechanical and Durability Properties and Behaviour of Recycled Aggregates Concrete. Journal of Building Engineering, 6 (1,15), 1 - 16.

Gomes, P., Ulsen, C., Pereira, F., Quattrone, M., Angulo, S. (2015). Comminution and Sizing Processes of Concrete Block Waste as Recycled Aggregates. Waste Management, 45 (171), 171 - 179.

Gursel, P. A., Masanet, E., Horvath, A., Stadel, A. (2014). Life-cycle inventory analysis of concrete production: A critical review. Cement \& Concrete Composites, 51 (45) 48 -58 .

Jian B., Sun, C., Jun, C. (2016). Materials Characteristics Affecting CO2 Curing of Concrete Blocks Containing Recycled Aggregates. Cement and Concrete Composites, 67 (50), $50-59$.

Kleijer, A., Lasvauxa, S., Citherlet, S., Vivianib, M. (2017). Product-specific Life Cycle Assessment of ready mix concrete: Comparison between a recycled and an ordinary concrete. Resources, Conservation and Recycling, 122 (217) 210 - 218.

López, F., González, J., López, C., Serrano, M., López, A. (2016). Life Cycle Assessment for Concrete Kerbs Manufactured with Recycled Aggregates. Journal of Cleaner Production, 113 (41, 52), $41-53$.

Lotfi, S., Eggimann, M., Wagner, E., Mroz, R., Deja J. (2015). Performance of Recycled Aggregate Concrete Based on a New Concrete Recycling Technology. Construction and Building Materials, 95 0, 243 - 256.

Marinković S. B., Malesev M., Ignjatović I. (2015). Life Cycle Assessment (LCA) of Concrete Made Using Recycled Concrete or Natural Aggregates. Universidad de Belgrado. República de Serbia.

Martínez-Soto, I.E., \& Mendoza-Escobedo, C.J. (2006). Comportamiento Mecánico de Concreto Fabricado con Agregados Reciclados. Ingeniería, investigación y tecnología, 7(3), 151-164. Recuperado en 19 de diciembre de 2015, de http:/ / www.scielo.org.mx/scielo.php?script=sci_arttext\&pid=S1405-

$77432006000300002 \& \operatorname{lng}=$ es\&tlng $=$ es.

Medina, C., Zhu, W., Howind, T., Sánchez, M., Frías, M. (2014). Influence of Mixed Recycled Aggregate on the Physical - Mechanical Properties of Recycled Concrete. Journal of Cleaner Production, 68 (216), 216 - 225.

Omary, S., Ghorbel, E., Wardeh, G. (2016). Relationships Between Recycled Concrete Aggregates Characteristics and Recycled Aggregates Concretes Properties. Construction and Building Materials, 108 (163,173), 163 - 174.

Rafaela Cardoso, R., Vasco, R., Brito, J., Dhir, R. (2015). Use of Recycled Aggregates from Construction and Demolition Waste in Geotechnical Applications: A Literature Review. Waste Management. 
Rodríguez, D., Luiz, J., Zanellato, F. (2016). Life cycle assessment (LCA) applied to the manufacturing of common and ecological concrete: A review. Construction and Building Materials, 124 (664), 656 - 666.

Saiz, P., Gonzalez, M., Fernandez, F., Rodríguez, A. (2015). Comparative Study of Three Types of Fine Recycled Aggregates from Construction and Demolition Waste (CDW), and Their Use in Masonry Mortar Fabrication. Journal of Cleaner Production, 118 (162), 162 - 169.

Sebben, R., Grigore, B., Hoffmann, C., Otavio C., Oliveira, R., Huchet, F. (2015). A Sorting Method to Value Recycled Concrete. Journal of Cleaner Production, 112 (2249), $2249-2258$.

Shi, C., Li, Y., Zhang, J., Li, W., Chong, L., Xie, Z. (2015). Performance Enhancement of Recycled Concrete Aggregate - A Review. Journal of Cleaner Production, 112 (466), $466-472$.

Silva, C. y Pereira P. (2015). Use of Recycled Construction and Demolition Materials in Geotechnical Applications: A Review. Resources, Conservation and Recycling, 103 (192), $192-204$.

Serres N., Braymand S. y Feugeas F. (2015). Environmental evaluation of concrete made from recycled concrete aggregate implementing Life Cycle Assessment, Journal of Building Engineering. http://dx.doi.org/10.1016/j.jobe.2015.11.004.

Stafford, F., Pereira, F., Labrincha J., b, Hotza, D. (2016). Life cycle assessment of the production of cement: A Brazilian case study. Journal of Cleaner Production, 137 (1298) $1293-1299$.

Turk, J., Cotic, Z., Mladenovic, A., Šajna, A. (2015). Environmental Evaluation of Green Concretes Versus Conventional Concrete by Means of LCA. Waste Management, 45 (194), $194-205$

Uzzal, M., Sun, C., Lob, I., Cheng, J. (2016). Comparative Environmental Evaluation of Aggregate Production from Recycled Waste Materials and Virgin Sources by LCA. Resources, Conservation and Recycling, 109 (67), 67 - 77.

\section{CITAR COMO:}

Elías Silupu, J. W., Flores Franco, J. E., Barrera Gutiérrez, R. E., \& Reyna Pary, C. A. (2020). Efecto de la Utilización de Agregados de Concreto Reciclado sobre el Ambiente y la Construcción de Viviendas en la Ciudad de Huamachuco. Puriq, 2(1), 16-27. https://doi.org/10.37073/puriq.2.1.68 